\title{
Ophthalmology and Social Media: An In-Depth
Investigation of Ophthalmologic Content on Instagram
}

\author{
Andy S Huang $\mathbb{D}^{\prime}$, Ali Adel Ne'ma Abdullah², Kelsey Chen ${ }^{3}$, Dagny Zhu $\mathbb{D}^{4}$ \\ 'Medical College of Georgia, Augusta, GA, USA; ${ }^{2}$ St Helier Hospital, Sutton, London, UK; ${ }^{3}$ Midwestern University, Glendale, AZ, USA; ${ }^{4}$ Hyperspeed \\ LASIK/NVISION Eye Centers, Rowland Heights, CA, USA \\ Correspondence: Andy S Huang, Tel +I 678 314 0208, Email andyhopkinshuang@gmail.com
}

Purpose: Social media has become a popular source of health information for patients. This study aimed to characterize the topperforming ophthalmologic posts on a large social media platform to better understand the spread of ophthalmic information via social media.

Materials and Methods: This was a web-based study that searched for ophthalmology-related posts on Instagram, with subjects being users who posted ophthalmic content. A list of 36 ophthalmology-related hashtags, including the most common diagnoses and procedures identified from the IRIS Registry, was queried. For each hashtag, data were collected for "Top 9 posts" (as ranked by Instagram's engagement-based algorithm) at three different time points. Posts were analyzed for the poster's background, credentials, post format, content, caption length, and engagement level.

Results: Of the top-performing posts analyzed $(\mathrm{n}=972)$, the most frequent post format was a photo $(82.2 \%)$, followed by video $(8.8 \%)$ and graphic (8.4\%). Ophthalmologists $(35.8 \%)$ authored the highest number of posts, followed by patients $(27.1 \%)$, optometrists (20.1\%), and organizations (12.7\%). The highest average engagement level ratios (ELRs) belonged to ophthalmologists-in-training (0.096), followed by patients $(0.084)$, optometrists $(0.070)$, all ophthalmologists $(0.067)$ and organizations $(0.051) ; p<0.001$. The most engaging type of content was self-promotional (0.118) and personal experience-related (0.091); educational content was the least engaging (0.059) even though it comprised the majority of posts $(56 \%)$; $(\mathrm{p}<0.001)$. Characteristics that predicted the highest ELRs (reaching 80th percentile) were captions and/or images that featured personal experiences (3.335 OR), whitecoats (3.259), and those authored by ophthalmologist trainees $(3.172)$; $(\mathrm{p}<0.01)$. The least engaging were those featuring fundus photos $(0.281)$, educational content (0.359), and authored by organizations $(0.428) ;(\mathrm{p}<0.05)$.

Conclusion: The majority of ophthalmologic content on Instagram is authored by non-ophthalmologists, with educational content being the least engaging. Practicing ophthalmologists have an opportunity to reach more patients through social media by incorporating specific features known to drive post engagement and reach.

Precis: Social media has become a popular source of health information for patients. Our study demonstrates that the majority of ophthalmology content on Instagram is authored by non- ophthalmologists, with educational content being the least engaging. Practicing ophthalmologists have an opportunity to reach a wider audience through social media by incorporating specific features known to drive post engagement and reach.

Keywords: ophthalmology, social media, Instagram, education, engagement, content, optometrist, ophthalmologist, patient, hashtag, engagement level ratio

\section{Introduction}

Never before in history has health information been disseminated at such a rapid rate as through the modern usage of the internet and social media. Social media encompasses various internet-based networks that allow individual patients, providers, and organizations to interact with each other in a multitude of ways. ${ }^{1}$ Social media platforms such as Facebook, Twitter, TikTok, and Instagram, have become one of the primary means of exchanging information with 
4.2 billion active global social media users worldwide as of Feb $25,2021 .^{2}$ Social media users have doubled within the last four years, with a significant boost due to the recent COVID pandemic.

In recent years, social media has become of ever-increasing importance for the field of healthcare, particularly with the public fear and desire to learn more about COVID-19. ${ }^{3}$ A study in 2020 demonstrated that the internet was the most frequently used source of health information, with the percentage of the population who used the internet first for their most recent search increasing from $61.2 \%$ in 2008 to $74.4 \%$ in $2017 .{ }^{4}$ An earlier study from the Pew Research Center's Internet and American Life Project found that $73 \%$ of American adults seek medical information from the internet; this figure will only increase as new technologies provide access to health information in unprecedented ways. ${ }^{5}$ During their search for medical information, individuals are also more likely to come across pages and profiles of medical professionals, organizations, and academic programs who are expanding their social media usage with each passing year. ${ }^{6}$

Ophthalmology is a highly visual specialty, allowing eye-related discussions to be more easily shared on image-based public platforms such as Instagram, which is being used by $40 \%$ of the US adult population. ${ }^{7}$ It is therefore unsurprising that Instagram contains a wide breadth of ophthalmic information and features the growing presence of professional organizations such as the American Academy of Ophthalmology (AAO), with over 30,000 followers and 256 posts. The purpose of this study was to characterize ophthalmology-related content on Instagram and to identify features associated with the top-performing ophthalmologic posts.

\section{Materials and Methods}

\section{Study Design and Study Population}

A list of 37 ophthalmology-related hashtags was queried on Instagram. Hashtags are keywords used to label individual posts. The terms were collected from the American Academy of Ophthalmology Intelligent Research in Sight (IRIS) Registry by most common ophthalmic conditions and procedures distributed across all subspecialties. General laymen's terms for ophthalmology were also included to find eye-related content. Hashtag terms that yielded few or irrelevant posts were excluded. One example was PinkEye, which yielded an overwhelming majority of makeup-related posts and was removed from the analysis, leaving a total of 36 hashtags. The full list of hashtag terms queried along with the total number posts associated with each are shown in Table 1.

For each hashtag, data were collected for the "Top 9 posts" ranked by Instagram at three time points from September 2020 to March 2021 as determined by Instagram's engagement level-based algorithm ( $\mathrm{n}=$ total 27 posts per hashtag). The Instagram algorithm prioritizes and ranks posts based on the number of likes and comments over a span of time, giving high-ranking posts greater reach. Duplicate posts, content unrelated to ophthalmology, and ophthalmic veterinary posts were excluded. A detailed methodology diagram is shown in Figure 1.

Each post was analyzed for the poster's background (eg, ophthalmologist, optometrist, patient, or organization), credentials (eg, ophthalmologist trainee, board-certified, non-board certified or international) as well as the post format (eg, photo, graphic, or video), content (eg, education, self-promotion, or personal experience), caption length, and engagement level ratios (ELR). ELRs were calculated as a ratio of number of post likes to account followers to aid in the comparison of posts. "Likes" refers to the number of accounts that "liked" the post, and "followers" refers to the number of accounts subscribed to the profile to receive regular updates on their feed.

Educational content was identified as posts focused primarily on teaching and/or increasing public awareness about specific ophthalmologic conditions or procedures. Examples included question/answer posts, slit lamp photos, fundus images, or videos of specific ophthalmic procedures. Self-promotional posts were those that primarily made references to the author's practice and/or advertised the procedures their practice could perform. Personal experience-related content encompassed posts that included subjective or historical narratives specific to the individual. Two examples are as follows: 1) a physician that shares their experiences with treating a specific ophthalmic condition and his/her challenges, 2) a patient that shares his/her perspective and/or journey with living with an ophthalmic condition (eg, strabismus, glaucoma, or retinal detachments). When considering caption length, the posts were separated into three groups: short (1-8 lines), medium (9-19 lines), and long ( $\geq 20$ lines). 
Table I List of Hashtag Terms Queried

\begin{tabular}{|c|c|}
\hline Hashtag Terms Queried & Total Posts \\
\hline \#Ophthalmology & $\mathrm{I}, 078,100$ \\
\hline \#Glaucoma & 697,000 \\
\hline \#Blepharoplasty & 674,400 \\
\hline \#LASIK & $5,57,900$ \\
\hline \#Ophthalmologist & 442,600 \\
\hline \#EyeSurgery & 367,100 \\
\hline \#Cataract & 303,200 \\
\hline \#CataractSurgery & 180,500 \\
\hline \#LASIKSurgery & 177,800 \\
\hline \#Муоріа & 169,800 \\
\hline \#DryEyes & 146,600 \\
\hline \#DryEye & $\mid 12,800$ \\
\hline \#Oculoplastics & 97,000 \\
\hline \#LaserEyeSurgery & 95,000 \\
\hline \#Ptosis & 71,900 \\
\hline \#MacularDegeneration & 69,300 \\
\hline \#StrabismusSurgery & 69,000 \\
\hline \#Strabismus & 64,600 \\
\hline \#Conjunctivitis & 56,800 \\
\hline \#EyeDisease & 50,300 \\
\hline \#DiabeticRetinopathy & 39,700 \\
\hline \#Pterygium & 33,200 \\
\hline \#DryEyeSyndrome & 28,200 \\
\hline \#PterygiumSurgery & 23,300 \\
\hline \#RetinalDetachment & 22,526 \\
\hline \#CornealTransplant & 21,500 \\
\hline \#Vitrectomy & 16,900 \\
\hline \#CornealUlcer & 15,500 \\
\hline \#CornealAbrasion & 15,000 \\
\hline \#Chalazion & 11,000 \\
\hline \#GlaucomaSurgery & 7000 \\
\hline \#RetinaSurgery & 7000 \\
\hline \#Keratitis & 4800 \\
\hline
\end{tabular}

(Continued) 
Table I (Continued).

\begin{tabular}{|l|c|}
\hline Hashtag Terms Queried & Total Posts \\
\hline \#ARMD & 3000 \\
\hline \#PRKSurgery & 3000 \\
\hline \#Trabeculoplasty & 1500 \\
\hline \#OrbitalCellulitis & 300 \\
\hline
\end{tabular}

Notes: Total posts captured is listed for each keyword. Hashtags are based on AAO's IRIS registry and general laymen's terms to broadly identify ophthalmic content.

Abbreviations: AAO, American Academy of Ophthalmology; IRIS, Intelligent Research in Sight.

\section{Statistical Analysis}

One-way ANOVA analyses were performed to compare and identify statistically significant differences of mean ELRs between different groups (eg, content, credentials, backgrounds, and caption lengths). The significance level $\alpha$ was set at 0.05. Odds Ratio (OR) and $95 \%$ confidence interval (CI) were calculated to predict the likelihood of posts with specific features (eg, slit lamp, fundus, "selfie", whitecoat, scrubs, and/or presence of ophthalmologist or optometrist within the picture) to reach the top $20 \%$ engagement levels ( $\geq 80$ th percentile ELR) of all posts within our inclusion criteria.

The American Board of Medical Specialties website (certificationmatters.org) was used to determine the boardcertification status of the ophthalmologists that had posted and were selected from our inclusion criteria. As Instagram data is publicly accessible, this study was exempt from the Institutional Review Board (IRB). This research adhered to the tenets of the Declaration of Helsinki.

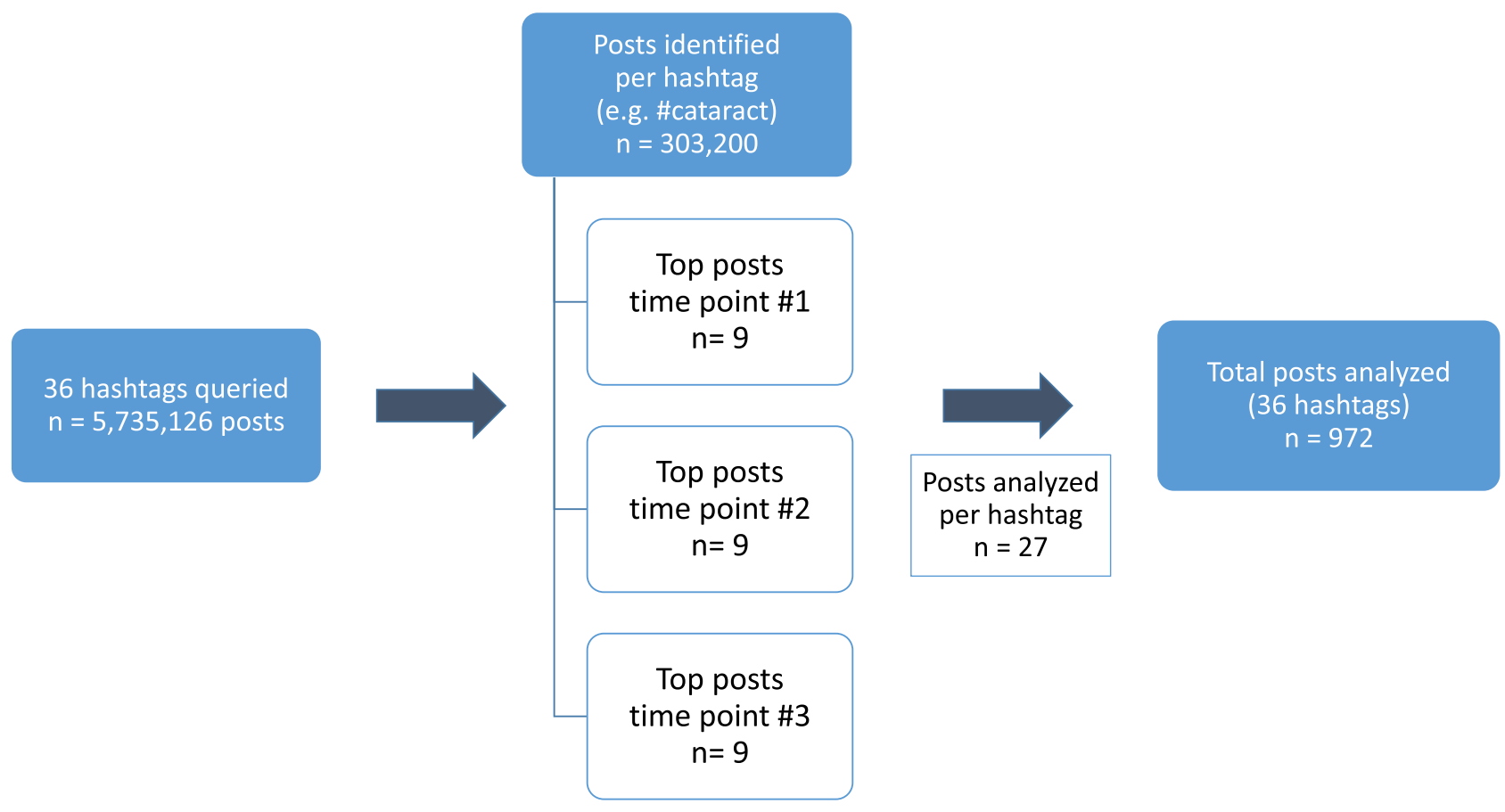

Figure I Methodology diagram for extraction of posts.

Notes: A search of 36 ophthalmic hashtags resulted in 5,735, 126 posts. IG's "Top 9 posts" for each hashtag were extracted from each of the three different time points for a total of 27 posts per hashtag. A total of 972 posts were analyzed.

Abbreviation: IG, Instagram. 


\section{Results}

A total of over 5.7 million Instagram posts were tagged using the 36 hashtags queried, of which 972 met the inclusion criteria for further analysis.

Ophthalmologists, optometrists, organizations, and patients constituted the majority of post authors $(95.6 \%, 929$ of 972). Ophthalmologists (35.8\%) authored the highest number of posts, followed by patients (27.1\%), optometrists (20.1\%), organizations (12.7\%), and others (4.4\%) (Figure 2). Ophthalmologists comprised of US/EU board-certified physicians $(50.9 \%)$, international/non-board-certified physicians $(33.6 \%)$, and trainees $(13.5 \%)$. The most frequent post format was a photo $(82.2 \%)$, followed by video $(8.8 \%)$ and graphic $(8.4 \%)$. In terms of content, educational posts $(56.3 \%)$ made up the majority of posts, followed by personal experience-related (34.0\%) and self-promotional $(9.3 \%)$.

When analyzing ELRs by the post author (Figure 3), the highest average engagement ratio belonged to ophthalmologists-in-training $(0.096)$, followed by patients $(0.084)$, optometrists $(0.070)$, all ophthalmologists $(0.067)$, and organizations (0.051); $\mathrm{p}<0.001$. International and non-board-certified ophthalmologists had an ELR of 0.062 despite constituting $33.6 \%$ of all posts analyzed.

In regards to content, the highest ELR belonged to posts related to self-promotion (0.118) and personal experiences (0.091); educational content garnered the lowest engagement levels $(0.059)$; $(\mathrm{p}<0.001)$. When analyzing by post format, videos yielded significantly higher engagement levels (ELR 0.110) compared to photos (ELR 0.073) and graphics (ELR 0.063) ( $\mathrm{p}=0.036$ ).

Table 2 shows the odds ratio (OR) of specific post features associated with ranking in the top $\geq 80$ th percentile or top 200 posts for engagement levels. The highest ORs belonged to posts exhibiting personal experiences (OR 3.335),

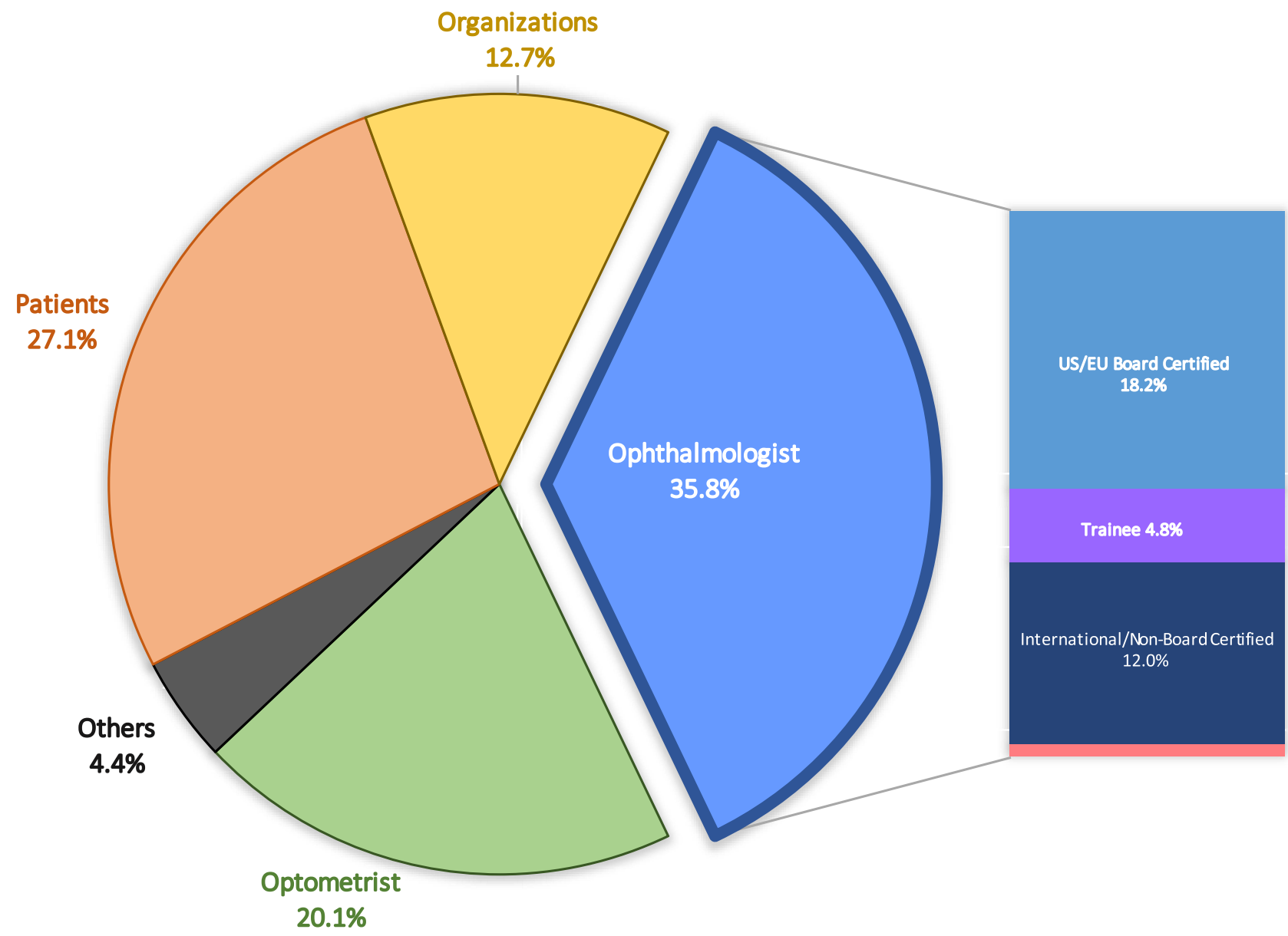

Figure 2 Breakdown of authors of ophthalmology-related content.

Note: Of all posters, all ophthalmologists consisted of $35.8 \%$, US/EU board-certified consisted of $18.2 \%$, international/non-board-certified ophthalmologists consisted of $12.0 \%$ and ophthalmic trainees consisted of $4.8 \%$. 


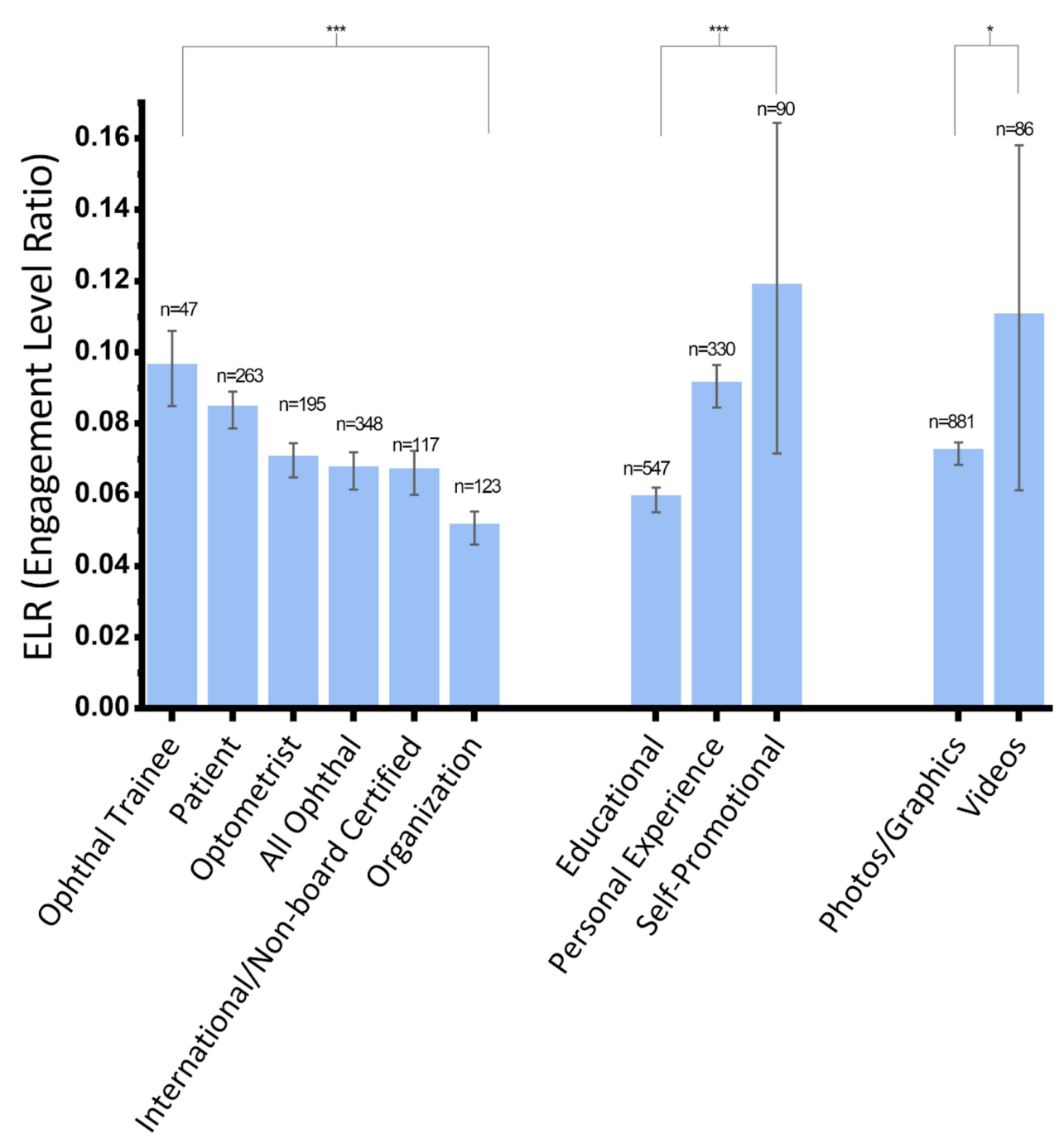

Figure 3 Engagement level ratio of author, post format, and content.

Notes: Authors of ophthalmology-related content Instagram, post format, and content and their impact on their average engagement level ratios (number of likes \% number of followers). Standard error of mean represented in gray. Note: $*_{p}<0.05 ; *^{*} *_{p}<0.001$.

whitecoats (3.259), trainee authors (3.172), and "selfies" (2.815); $(\mathrm{p}<0.01)$. The lowest ORs belonged to posts displaying fundus images (0.281), educational content (0.359), organization authors (0.428), and board-certified ophthalmologist authors (0.4825); $(\mathrm{p}<0.05)$. "Selfie" posts, defined as a close-up photo of one or several individuals, made up most of the specific visuals analyzed (24.2\%). Of the selfies analyzed, $71.4 \%$ of them showcased the patient, with the remainder belonging to ophthalmologists, optometrists, or other individuals. When analyzing caption length, the posts with the highest ORs were short $(1.551, \mathrm{p}<0.01)$, with long captions coming in last $(0.700, \mathrm{p}<0.05)$.

\section{Discussion}

Social media platforms such as Instagram contain a wide breadth of ophthalmic information and continue to grow rapidly. This new avenue allows ophthalmologists to engage with audiences in an unprecedented manner, allowing for communication with potentially hundreds of thousands of individuals on a more rapid and profound scale than was ever possible with traditional methods. This is mainly seen as a positive change, as communication between patients and healthcare professionals on Instagram can allow for a more harmonious relationship through patient empowerment, equalized and reciprocal communication, and improved self-management, all of which can lead to better clinical decision-making. ${ }^{8}$ 
Table 2 Odds Ratio Analysis of Factors in Ophthalmic Instagram Posts

\begin{tabular}{|c|c|c|c|c|}
\hline & Factors of Ophthalmic IG Posts & OR for 80th Percentile & Lower $95 \% \mathrm{Cl}$ & Upper $95 \% \mathrm{Cl}$ \\
\hline \multirow{7}{*}{ Author of post } & Trainee & $3.172^{* * *}$ & 1.444 & 6.97 \\
\hline & Patient & 2. $104 * * *$ & 1.516 & 2.921 \\
\hline & Optometrist & 0.926 & 0.627 & 1.366 \\
\hline & Ophthalmologist & 0.737 & 0.529 & 1.028 \\
\hline & Non-board Certified & 0.637 & 0.375 & 1.081 \\
\hline & Board Certified & $0.483 * *$ & 0.262 & 0.9 \\
\hline & Organization & $0.428 * *$ & 0.24 & 0.765 \\
\hline \multirow{3}{*}{ Post Format } & Photo & $3.157 * * *$ & 1.807 & 5.516 \\
\hline & Graphics & 0.651 & 0.352 & 1.204 \\
\hline & Video & $0.442^{*}$ & 0.224 & 0.872 \\
\hline \multirow{3}{*}{ Post Content } & Personal Experience & $3.335 * * *$ & 2.408 & 4.621 \\
\hline & Self-Promotion & $\mathrm{I} .077$ & 0.632 & 1.835 \\
\hline & Educational & $0.359 * * *$ & 0.259 & 0.498 \\
\hline \multirow{7}{*}{ Specific Visuals } & White Coat & $3.259 * *$ & 1.456 & 7.294 \\
\hline & Selfie & $2.815 * * *$ & 2.021 & 3.202 \\
\hline & Optometrist in content & $2.452^{*}$ & 1.213 & 4.958 \\
\hline & Scrubs & $2.28 I^{*}$ & 1.036 & 5.021 \\
\hline & Ophthalmologist in content & 1.238 & 0.801 & 1.913 \\
\hline & Slit Lamp & $0.538^{*}$ & 0.311 & 0.932 \\
\hline & Fundus & $0.28 I^{*}$ & 0.086 & 0.918 \\
\hline \multirow{3}{*}{ Caption Length } & Short & $1.551 * *$ & 1.124 & 2.141 \\
\hline & Medium & 1.066 & 0.765 & 1.485 \\
\hline & Long & $0.700 *$ & 0.492 & 0.995 \\
\hline
\end{tabular}

Notes: Odds Ratio Analysis to measure the association between various factors of ophthalmic Instagram posts and the outcome of the post being in the $\geq 80$ th percentile (or top 200 posts for ELR). For example, the odds of being in the top $20 \%$ of all ophthalmic Instagram within our analysis are 3.17 times higher in posts with white coats than posts that did not include white coats; $(\mathrm{p}<0.01)$. Note: $*_{p}<0.05$; ** $p<0.01$; *** $p<0.001$.

Abbreviations: IG, Instagram; OR, Odds ratio; $\mathrm{Cl}$, Confidence interval.

There have been similar studies in other specialties, including plastic surgery, dermatology, and obstetrics/gynecology, that have attempted to characterize social media post authors and content as well as analyze degrees of various interests (eg, types of surgeries or treatments plans), the prevalence of disease, and what is trending within a specialty. ${ }^{9-17}$ To our knowledge, this study is the first to have conducted such a broad analysis of ophthalmology content on Instagram.

\section{Related Studies of Social Media Within Ophthalmology}

There is a tremendous increase in the popularity of social media use by ophthalmologists for educating and disseminating eye health initiatives with patients, colleagues, and the academic community. ${ }^{18}$ In a recent survey, $72 \%$ and nearly $40 \%$ of ophthalmologists reported using social media for personal and professional purposes, respectively. ${ }^{19}$ With this rise, several studies have analyzed social media usage amongst patients and healthcare professionals. Clarke et al analyzed patient social media usage during the LASIK surgery perioperative period. ${ }^{20}$ Park et al analyzed the most and least 
successful Instagram posts of oculoplastic surgeons and demonstrated that engagement increased when featuring the posing doctor smiling and wearing a white coat, whereas posts depicting procedures had less engagement. ${ }^{21}$ These findings were similar to our study's results demonstrating higher engagement levels amongst "selfies" and posts showcasing the physician wearing a white coat. McGregor et al evaluated social media use by glaucoma patients and demonstrated commonly discussed topics including treatments, care experiences, and support. ${ }^{22}$ They also highlighted an opportunity for ophthalmologists to promote therapies and treatments with more established evidence to combat misinformation. ${ }^{22}$ Micieli and Tsui (2015) evaluated ophthalmology organization involvement on Facebook, Twitter, and LinkedIn and concluded that there is an opportunity for these organizations to increase their presence and involvement on social media. ${ }^{23}$ Micieli and Micieli (2012) reached a similar conclusion when analyzing ophthalmologist usage of Twitter. ${ }^{24}$ Johnson et al assessed the content, intent, and authorship of the vision therapy-related Instagram hashtags within pediatric ophthalmology and found that the majority of posts were self-promotional and advertisements, and that of 1766 posts analyzed, surprisingly only $14(0.8 \%)$ were made by physicians. ${ }^{25}$ These studies illustrate a need for greater content creation by practicing ophthalmologists on social media. While our study findings revealed a relatively high proportion of ophthalmologist-authored posts and educational content, these posts were overall lowperforming and demonstrate the need for the creation of more engaging content in order to reach a greater number of patients.

\section{Highlights and Implications of Study Findings}

To our knowledge, our study is the first to conduct a broad and comprehensive analysis of ophthalmology content on Instagram by utilizing a wide range of ophthalmology-related hashtag terms across multiple sub-specialties. A particularly unique aspect was analyzing specific post features and characteristics to assess its impact on engagement levels and overall post-performance and reach. Our results show that the performance of posts varies drastically based on the post author, format, content, visuals, and caption length.

While ophthalmologists represented the largest group of post creators, the majority of ophthalmology content on Instagram was created by non-ophthalmologists. This is in accordance with studies in other fields (dermatology, plastic surgery, and urogynecology) that have shown that the majority of top social media content is created by individuals without formal board-certified training. ${ }^{10,13,14,25}$

In addition, post authorship by a board-certified ophthalmologist was more likely to be associated with lower engagement levels (the 5th lowest of the 23 specific factors studied). Thus, there is an opportunity for practicing ophthalmologists to create more engaging content when compared to ophthalmologists-in-training, optometrists, and patients. Similarly, while educational content was the most abundant of all content types, posts containing educational material achieved the lowest engagement levels, far behind self-promotion and personal experiences. Traditional slitlamp photos, fundus photos, videos on surgical techniques, and infographics performed with the lowest engagement levels. While such educational content is important to share, low-performing posts are often hidden and buried by engagement-based algorithms used by social media platforms and are thus seen by a much smaller audience. Again, there is a clear opportunity for ophthalmologists, particularly board-certified ophthalmologists, to reach a broader audience by producing more engaging educational content, perhaps by incorporating some factors that were demonstrated to be positively associated with higher engagement in our study (eg, shorter captions, "selfie" photos, scrubs or whitecoat attire, etc.). In addition, because personal experience-related content performed better, ophthalmologists may consider adding a more personal touch to their educational content by sharing unique perspectives, experiences, and/or reflections in order to increase post engagement and reach.

\section{Limitations}

There are several limitations to our study. Firstly, the high levels of engagement for self-promotional posts may have been skewed by paid advertising promotions through Instagram artificially increasing likes. However, all advertisement posts are labeled as such, and very few were found in this study. Additionally, there are two discrepancies between the ANOVA ELR analysis and OR analysis for $\geq 80$ th percentile. Although self-promotional posts and videos performed best in ELR averages, they scored lower than expected in the OR analysis. This may have been due to the volume of low- 
performing non-videos and personal experience posts that skewed the overall mean downward. Therefore, the OR data was prioritized in our interpretation of our data. Finally, social media is fluid, and the ranking of posts due to modifications of engagement-based algorithms is constantly changing. Our study captures only a specific snapshot in time and may not accurately represent social media ophthalmology content in the future.

In this modern digital age, social media has become an exciting and invaluable tool for sharing health information for medical professionals and patients alike. However, as social media content is not regulated nor always evidence-based, online health information needs to be shared responsibly and evaluated with utmost caution. As such, practicing ophthalmologists have an exciting opportunity and responsibility to contribute accurate, relevant, and engaging ophthalmologic content in today's growing digital era.

\section{Funding}

There is no funding to report.

\section{Disclosure}

The authors declare that they have no conflicts of interest in this work.

\section{References}

1. Carr CT, Hayes RA. Social media: defining, developing, and divining. Atl J Commun. 2015;23(1):46-65. doi:10.1080/15456870.2015.972282

2. Statista Research Department. Social media - statistics \& facts [Internet]. Statista. 2021. Available from: https://www.statista.com/topics/1164/ social-networks/.

3. Li C, Chen LJ, Chen X, Zhang M, Pang CP, Chen H. Retrospective analysis of the possibility of predicting the COVID-19 outbreak from Internet searches and social media data, China, 2020. Euro Surveill. 2020;25(10):2000199. doi:10.2807/1560-7917.ES.2020.25.10.2000199

4. Finney Rutten LJ, Blake KD, Greenberg-Worisek AJ, Allen SV, Moser RP, Hesse BW. Online health information seeking among US adults: measuring progress toward a healthy people 2020 objective. Public Health Rep. 2019;134(6):617-625. doi:10.1177/0033354919874074

5. Lenhart A, Purcell K, Smith A, Zickuhr K. Social media and young adults [Internet]. Pew Research Center. 2010. Available from: https://www. pewresearch.org/internet/2010/02/03/social-media-and-young-adults/.

6. Tsui E, Rao RC. Navigating social media. Ophthalmology. 2019;126(6):779-782. doi:10.1016/j.ophtha.2019.02.015

7. Auxier B, Anderson M. Social media use in 2021 [Internet]. Pew Research Center. 2021. Available from: https://www.pewresearch.org/internet/ 2021/04/07/social-media-use-in-2021/.

8. Smailhodzic E, Hooijsma W, Boonstra A, Langley DJ. Social media use in healthcare: a systematic review of effects on patients and on their relationship with healthcare professionals. BMC Health Serv Res. 2016;16(1):442. doi:10.1186/s12913-016-1691-0

9. Braun SE, O'Connor MK, Hornick MM, Cullom ME, Butterworth JA. Global trends in plastic surgery on social media: analysis of 2 million posts. Aesthet Surg J. 2021;41(11):1323-1332. doi:10.1093/asj/sjab185

10. Dorfman RG, Vaca EE, Mahmood E, Fine NA, Schierle CF. Plastic surgery-related hashtag utilization on Instagram: implications for education and marketing. Aesthet Surg J. 2018;38(3):332-338. doi:10.1093/asj/sjx120

11. Mullens CL, Hardy KM, Hernandez JA, et al. PlasticSurgery: a comparative deep dive analysis into social media and plastic surgery. Plast Reconstr Surg. 2020;146(2):413-422. doi:10.1097/PRS.0000000000007001

12. Braunberger T, Mounessa J, Rudningen K, Dunnick CA, Dellavalle RP. Global skin diseases on Instagram hashtags. Dermatol Online J. 2017;23 (5):13030/qt7sk410j3. doi:10.5070/D3235034925

13. Ranpariya V, Chu B, Fathy R, Lipoff JB. Dermatology without dermatologists? Analyzing Instagram influencers with dermatology-related hashtags. J Am Acad Dermatol. 2020;83(6):1840-1842. doi:10.1016/j.jaad.2020.05.039

14. Qin LA, El-Neemany D, Winkler H, Shalom D. Urogyn: what's trending on Instagram? A cross-sectional observational study. Female Pelvic Med Reconstr Surg. 2020;26(5):283-286. doi:10.1097/SPV.0000000000000869

15. Peyser A, Goldstein L, Mullin C, Goldman RH. Fertility education: what's trending on Instagram. Fertil Res Pract. 2021;18(7):3. doi:10.1186/ s40738-021-00095-6

16. Sinha R, Shibata R, Patel A, Sternchos JA. Social media in minimally invasive gynecologic surgery: what is trending on Instagram? J Minim Invasive Gynecol. 2021;28(10):1730-1734. doi:10.1016/j.jmig.2021.02.011

17. Park SSE, Akella SS, Moon J-Y, et al. Building your brand: analysis of successful oculoplastic surgeons on social media. Ophthalmic Plast Reconstr Surg. 2020;36(6):582-589. doi:10.1097/IOP.0000000000001654

18. Men M, Fung SSM, Tsui E. What's trending: a review of social media in ophthalmology. Curr Opin Ophthalmol. 2021;32(4):324-330. doi:10.1097/ICU.0000000000000772

19. Al-khersan H, Lazzarini TA, Fan KC, et al. Social media in ophthalmology: an analysis of use in the professional sphere. Health Informatics $J$. 2020;26(4):2967-2975. doi:10.1177/1460458220954610

20. Clarke C, Smith E, Khan M, Al-Mohtaseb Z. Social media and ophthalmology: perspectives of patients and ophthalmologists. J Med Syst. 2018;42 (12):258. doi:10.1007/s10916-018-1079-2

21. Park JH, Christman MP, Linos E, Rieder EA. Dermatology on Instagram: an analysis of hashtags. J Drugs Dermatol. 2018;17(4):482-484.

22. McGregor F, Somner JEA, Bourne RR, Munn-Giddings C, Shah P, Cross V. Social media use by patients with glaucoma: what can we learn? Ophthalmic Physiol Opt. 2014;34(1):46-52. doi:10.1111/opo.12093 
23. Micieli JA, Tsui E. Ophthalmology on social networking sites: an observational study of Facebook, Twitter, and LinkedIn. Clin Ophthalmol. 2015;10(9):285-290.

24. Micieli R, Micieli JA. Twitter as a tool for ophthalmologists. Can J Ophthalmol. 2012;47(5):410-413. doi:10.1016/j.jcjo.2012.05.005

25. Johnson A, Khan S, Koo EB. Social media and vision therapy: perspectives of providers and patients on Instagram. J AAPOS. 2021;25(3):166.e1166.e5. doi:10.1016/j.jaapos.2020.11.023

\section{Publish your work in this journal}

Clinical Ophthalmology is an international, peer-reviewed journal covering all subspecialties within ophthalmology. Key topics include: Optometry; Visual science; Pharmacology and drug therapy in eye diseases; Basic Sciences; Primary and Secondary eye care; Patient Safety and Quality of Care Improvements. This journal is indexed on PubMed Central and CAS, and is the official journal of The Society of Clinical Ophthalmology (SCO). The manuscript management system is completely online and includes a very quick and fair peer-review system, which is all easy to use. Visit http://www. dovepress.com/testimonials.php to read real quotes from published authors.

Submit your manuscript here: https://www.dovepress.com/clinical-ophthalmology-journal 